\title{
Emission of particles and photons in the pulsar polar cap
}

\author{
A. I. Tsygan \\ Ioffe Physical Technical Institute, Politekhnicheskaya, 26, 194021 \\ St.Petersburg, Russia
}

\begin{abstract}
We study emission of particles and photons from a pulsar polar cap. The Goldreich-Julian model for the regime of free emission of charged particles from the neutron star surface is used. In this case the electric field is generated due to the general relativistic effect of dragging of inertial frames. The spectra and shapes of gamma-ray pulses, the parameters of the electron-positron plasma and the intensity of X-ray emission from hot spots in the polar region of radio pulsars are discussed. The effect of non-dipole magnetic field on $\mathrm{X}$-ray emission of polar caps is considered. It is shown that the increase of magnetic line curvature leads to much smaller temperatures and X-ray luminosities of the polar caps as compared with the purely dipole field.
\end{abstract}

\section{Introduction}

The electric fields of the rotating neutron stars (radio pulsars) with a dipole magnetic ficld (in the "free emission" regime of charged particles) is determined by the general relativistic effect of drag of inertial reference frames (Muslimov \& Tsygan 1990, 1991, 1992; Beskin 1990). A general relativistic electrodynamical model of radio pulsars with the dipole magnetic field was presented first by Muslimov and Tsygan at the IAU Colloquium No. 128 held in Lagow, Poland (1990). We generalized this model for an arbitrary axially symmetric magnetic field (Pal'shin \& Tsygan 1999).

Let us consider a rotating neutron star (NS) surrounded by a corotating magnetosphere, that was introduced in the physics of radio pulsars by Goldreich \& Julian (1969). Jones (1985) as well as Neuhauser, Koonin \& Langanke (1987) yield that the work function of ions and electrons for a NS surface is 200-300 $\mathrm{eV}$ (for the magnetic ficld $B=10^{12} \mathrm{G}$ ). For a $\mathrm{NS}$ with the surface temperature $T>2 \times 10^{5} \mathrm{~K}$, the thermal emission of electrons and ions is important.

\section{The electrodynamical model}

In the reference frame corotating with a NS the electrostatic potential in the quasistatic region of open field lines satisfies the Poisson equation modified by the effects of General Relativity (Muslimov \& Tsygan 1990, 1991, 1992):

$$
\nabla \cdot\left(\frac{1}{\alpha} \nabla \Phi\right)=-4 \pi\left(\rho+\rho_{e f f}\right)
$$




$$
\begin{gathered}
\rho_{e f f}=\frac{1}{4 \pi} \nabla \cdot(\alpha \vec{g} \times \vec{B})=\frac{\alpha \vec{B}}{4 \pi} \nabla \times \vec{g}, \\
\vec{g}=\left(1 / c \alpha^{2}\right)\left[1-\kappa(a / r)^{3}\right] \vec{\Omega} \times \vec{r}, \\
\vec{E}=-\nabla \Phi ;\left.\Phi\right|_{s}=0 ;\left.(\vec{E} \cdot \vec{B})\right|_{r=a}=0 .
\end{gathered}
$$

Here $\rho_{\text {eff }}$ is the Goldreich-Julian charge density in General Relativity, $\rho$ is the particle charge density; $\alpha=\sqrt{1-r_{g} / r}$ is the gravitational redshift function, $r_{g}=2 G M / c^{2}$ is the gravitational radius of the NS (of mass $M$ ); $\kappa=$ $\left(r_{g} / a\right) \cdot\left(I / M a^{2}\right)=\varepsilon \cdot \beta=0.12-0.16, \varepsilon=r_{g} / a=0.3-0.4$ is the compactness parameter, $a$ is the stellar radius (more exactly, the radial coordinate of the surface) and $\beta=I / M a^{2}$ is the stellar moment of inertia in the units of $M a^{2}$. The differential operator is taken in three-dimensional curvilinear coordinates for the Schwarzschild metric.

Let us consider an arbitrary axially symmetric magnetic field and a thin tube of open field lines. In a small-angle approximation for the boundary condition at the surface and the tube bottom, $\left.\Phi\right|_{s}=0$, the electric field is determined by the local charge density $\left(\rho+\rho_{e f f}\right)$. For the "free emission" regime $\left(\left.\vec{E} \cdot \vec{B}\right|_{r=a}=0\right)$, the condition $\left.\left(\rho+\rho_{e f f}\right)\right|_{r=a}=0$ will be valid on the stellar surface $\left(\theta_{0} \ll 1\right)$. For a dipole magnetic field, it was found by Muslimov \& Tsygan (1992).

Let us adopt the small-angle approximation and use a spherical coordinate system with the polar axis directed along the magnetic axis. Then Eq. (1) takes the form:

$$
\begin{gathered}
\frac{\alpha}{\eta^{2}} \frac{\partial}{\partial \eta}\left(\eta^{2} \frac{\partial \Phi}{\partial \eta}\right)+\frac{1}{\alpha \eta^{2} \vartheta} \frac{\partial}{\partial \vartheta}\left(\vartheta \frac{\partial \Phi}{\partial \vartheta}\right)=-4 \pi a^{2}\left(\rho+\rho_{e f f}\right), \\
\rho_{e f f} \approx(\vec{\Omega} \cdot \vec{B} / 2 \pi \alpha c)\left(1-\kappa / \eta^{3}\right) \approx(\Omega B / 2 \pi \alpha c)\left(1-\kappa / \eta^{3}\right) \cos \chi,
\end{gathered}
$$

where $\chi$ is the angle between the magnetic and spin axes and $\eta=r / a$ is a distance from the star in the units of stellar radius. Taking into account the continuity equation $\nabla \cdot(\alpha \vec{j})=0,($ where $\vec{j}=\rho c \vec{B} / B)$, and the Maxwell equation $\nabla \cdot \vec{B}=0$, we obtain: $\vec{B} \cdot \nabla(\alpha \rho / B)=0$. The magnetic field is nearly radial and depends only on the angle $\vartheta$. Using the condition $\left.\left(\rho+\rho_{e f f}\right)\right|_{\eta=1}=0$, we can obtain $\alpha \rho / B$ as a function of $\vartheta$. The charge density of relativistic particles can be written approximately as

$$
\begin{gathered}
\rho \approx-(\Omega B / 2 \pi \alpha c)(1-\kappa) \cos \chi, \\
\rho+\rho_{e f f} \approx \kappa(\Omega B / 2 \pi \alpha c)\left(1-1 / \eta^{3}\right) \cos \chi .
\end{gathered}
$$

Along all the tube (excluding the region near the tube bottom) the first term on the left-hand side of Eq. (2) can be neglected. In this case the solution which satisfies the boundary conditions is:

$$
\Phi(\eta, \xi)=(\kappa / 2)(\Omega a / c) a \eta^{2} \theta^{2}(\eta) B(\eta)\left(1-1 / \eta^{3}\right)\left(1-\xi^{2}\right) \cos \chi
$$


For $\cos \chi=0$ we must use the results of Arons \& Scharlemann (1979) (or Muslimov \& Tsygan 1990, 1991, 1992 for Schwarzschild metric).

The coordinate $\xi \equiv \vartheta / \theta(\eta)$ describes position of a point across the cone of open magnetic ficld lines $(0 \leq \xi \leq 1)$, where $\theta(\eta)$ is the polar angle of the last open line. The solution (4) is valid for arbitrary magnetic field (in Schwarzschild metric) and was derived Pal'shin \& Tsygan (1999). The value $\theta(1)$ for $\cos \chi \neq$ 1 is determined by the angular velocity of the star and the structure of the magnetic field in the Schwarzschild metric. For $\cos \chi=1$, the angle $\theta(1)$ is determined by the energy density of particles accelerated by the stellar electric field. The value $\theta(1)$ may also depend on external conditions, for example, on the external magnetic field (Tsygan 1997).

Note the lucky circumstance that allows us to determine the function $B(\eta) \eta^{2} \theta^{2}(\eta)$. From $\nabla \cdot \vec{B}=0$ we obtain conservation of magnetic flux of the tube $\pi a^{2} \eta^{2} \theta^{2}(\eta) B(\eta)=F=$ const. The flux itself can be calculated at the light cylinder, where the field is determined by the NS magnetic moment:

$$
\begin{gathered}
F=\left.\int_{(2 \pi)} \vec{B}\right|_{r=r_{L}} \cdot \vec{n} d S=\frac{2 \pi m \Omega}{c}, \\
\eta^{2} \theta^{2}(\eta) B(\eta)=\left(2 m / a^{3}\right)(\Omega a / c)=\theta_{0}^{2} B_{0} .
\end{gathered}
$$

In this case $B_{0}=2 \mathrm{~m} / \mathrm{a}^{3}$ is the "effective" dipole magnetic field at the NS pole and $\theta_{0}=\sqrt{\Omega a / c}$ is the "effective" angle of open magnetic field lines. Then the electrostatic potential can be written in the form:

$$
\Phi(\eta, \xi)=(\kappa / 2)(\Omega a / c) a \theta_{0}^{2} B_{0}\left(1-1 / \eta^{3}\right)\left(1-\xi^{2}\right) \cos \chi .
$$

Clearly this equation remains valid also for the magnetic tubes whose axes are slightly bent.

\section{Gamma-ray and X-ray emission from pulsar polar caps}

The electrons emitted from the surface of the NS in the polar cap region are accelerated by the potential $\Phi$ along the magnetic field to the Lorentz factor $\gamma(\eta, \xi)=e \Phi(\eta, \xi) / m c^{2} \simeq 5 \times 10^{6}-5 \times 10^{7}$. While moving along the curved magnetic field lines, they emit gamma-quanta by the "curvature mechanism". The quanta, in their turn, create electron-positron pairs. The electron-positron plasma screens the longitudinal electric field at some altitude $\eta_{p}$, thereby producing the "gap" in which particle acceleration occurs (Sturrock 1971; Ruderman \& Sutherland 1975).

In this case Eq. (6) is valid (for $\left(\eta_{p}-1\right) \gg \theta\left(\eta_{p}\right)$ ) in the region $(1+\theta(1))<$ $\eta<\left(\eta_{p}-\theta\left(\eta_{p}\right)\right)$. Charged particles are separated in the region $1<\eta<\eta_{p}$. All positrons born in the "gap" are accelerated toward the stellar surface, while the secondary electrons are accelerated away from the star. The number density of the primary electrons is also changed. Let us estimate the charge density $\rho_{+}(1)$ of the returning positrons and the modified charge density $\rho(1)$ of primary electrons from compensation of longitudinal electric field at the both boundaries 
of the "gap":

$$
\begin{aligned}
\rho(1)+\rho_{+}(1)+\rho_{e f f}(1) & =0, \\
\rho\left(\eta_{p}\right)+\rho_{-}\left(\eta_{p}\right)+\rho_{e f f}\left(\eta_{p}\right) & =0 .
\end{aligned}
$$

From the condition $\alpha \rho / B=$ const $(\eta)$ we have $\alpha\left(\eta_{p}\right) \rho\left(\eta_{p}\right) / B\left(\eta_{p}\right)=\alpha(1) \rho(1) / B(1)$. In addition the equality of the currents of positrons at $\eta=1$ and electrons at $\eta=\eta_{p}$ may be fulfilled if $\rho_{+}(1) \alpha(1) \theta^{2}(1)=-\rho_{-}\left(\eta_{p}\right) \alpha\left(\eta_{p}\right) \eta_{p}^{2} \theta^{2}\left(\eta_{p}\right)$. The solution of $\mathrm{Eq} .(7)$ is:

$$
\begin{aligned}
\rho_{+}(1) & =[0.5 \kappa /(1-\kappa)]\left(1-1 / \eta_{p}^{3}\right) \rho_{e f f}(1) \\
\rho(1) & =-\left[1+0.5 \kappa\left(1-1 / \eta_{p}^{3}\right) /(1-\kappa)\right] \rho_{e f f}(1)
\end{aligned}
$$

Moreover, for simplicity, let us take into account only the terms linear with respect to the parameter $\varepsilon=r_{g} / a$ (or $\kappa$ ). It is sufficient to use $\mathrm{Eq}$. (6) for $\Phi$ and perform calculations in the flat space-time. The pulsar energy loss rate due to acceleration of charged particles at the polar caps is:

$$
L=2 c \rho(1) \theta^{2}(1) 2 \pi a^{2} \int \Phi\left(\eta_{p}, \xi\right) \xi d \xi=(3 \kappa / 2)\left(1-1 / \eta_{p}^{3}\right) L_{m} \cos ^{2} \chi
$$

where $L_{m}=(1 / 6) B_{0}^{2} a^{2} c(\Omega a / c)^{4}$.

Stopping of positrons in the surface layer of the NS will eventually lieat the surface. We can estimate the temperature of a point on the surface from the condition that the flux of warm X-ray emission be equal to the flux of positron energy: $\sigma T^{4}(\xi)=\rho_{+}(1) c \Phi\left(\eta_{p}, \xi\right)$. We will neglect the heat flux inside the star. Then the $\mathrm{X}$-ray luminosity of the hot spots is:

$$
L_{X} \approx\left(\rho_{+}(1) / \rho(1)\right) L=3\left[(\kappa / 2)\left(1-1 / \eta_{p}^{3}\right)\right]^{2} L_{m} \cos ^{2} \chi .
$$

For $\theta_{0}=\sqrt{\Omega a / c} \ll 1$, the potential $\Phi$, the energy loss rate $L$, and the $\mathrm{X}$-ray luminosity $L_{X}\left(\right.$ at $\left.1 / \eta_{p}^{3} \ll 1\right)$ depend only on the dipole component of the NS magnetic field. However, the magnetic anomalies affect the gamma-ray emission, the temperature of the hot spots, the multiplication factor of the electron-positron plasma, and the value of $\eta_{p}$. Let us present the examples of numerical calculations the heights of the "gap" $\left(z_{p}=\eta_{p}-1\right)$ and the temperatures as function $\xi$ for the purely dipole magnetic field (Fig. 1), and also the X-ray luminosities from pulsar polar caps (Fig. 3). This calculations was performed in case when the height of the "gap" is determined by the "curvature radiation". Fig. 2 shows the shape and the spectrum of gamma-pulse for PSR 1706-44 ( $P=0.102 \mathrm{~s}$, $B=6.2 \times 10^{12} \mathrm{G}, \chi=10^{\circ}$, the angle between rotational axis and line of sight is $12.6^{\circ}$ ) also for the purely dipole field. The results for non dipole field is shown on Fig. 4. We used the model, in which the magnetic field represents as sum of dipole field and additional magnetic moment $\vec{m}_{1} \perp \vec{m}$, that placed at the depth of $0.1 a$ from the surface in polar region of NS (Pal'shin \& T'sygan 1998). The parameter $\beta$ equal the ratio of the magnetic fields of dipoles $\overrightarrow{m_{1}}$ and $\vec{m}$.

The results of our calculations (Pal'shin \& Tsygan 1998, 1999) are slightly different from the results of Pal'shin \& Tsygan (1997), Muslimov \& Harding (1997) and Harding \& Muslimov (1998) and can explain the observed intensity of X-ray emission from the polar caps of radio pulsars. 


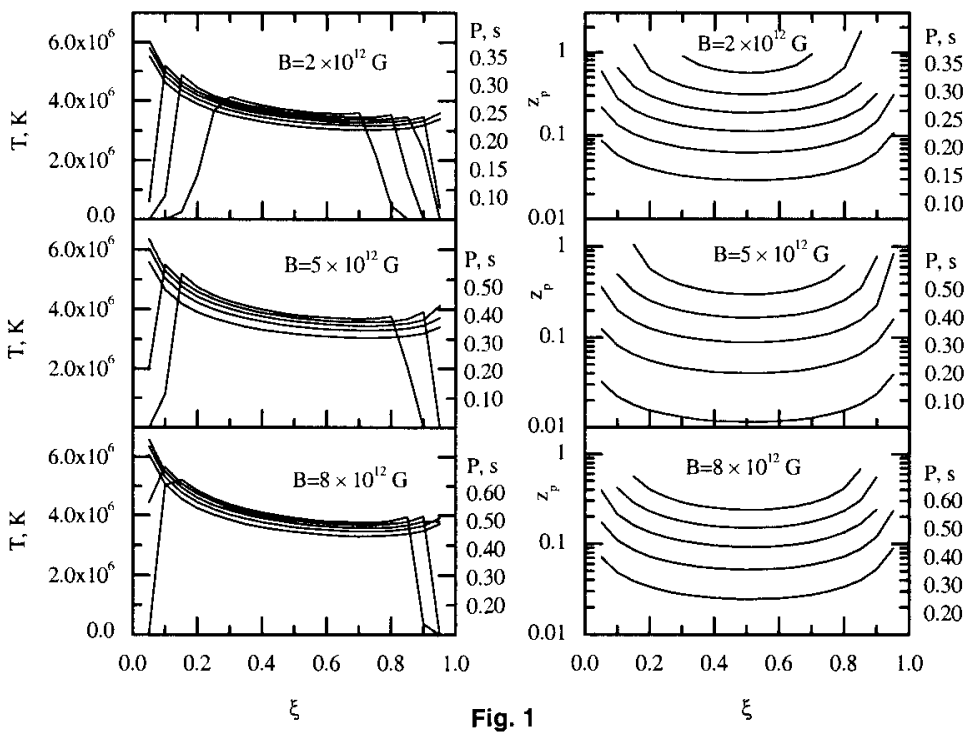

PSR 1706-44
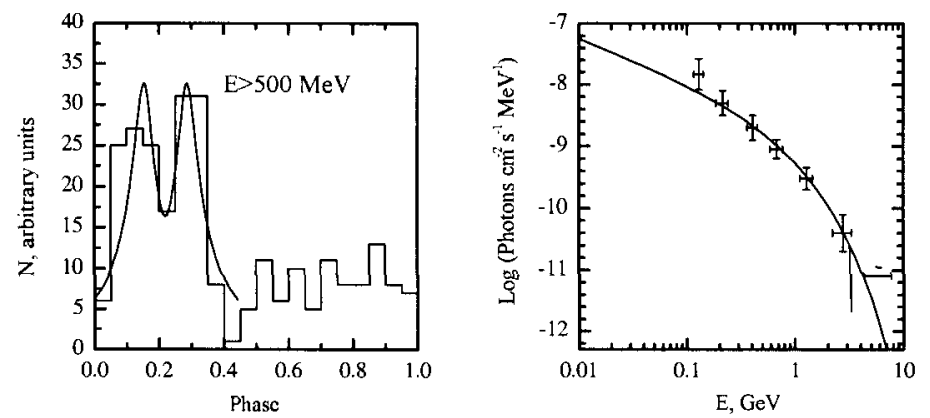

Fig. 2 


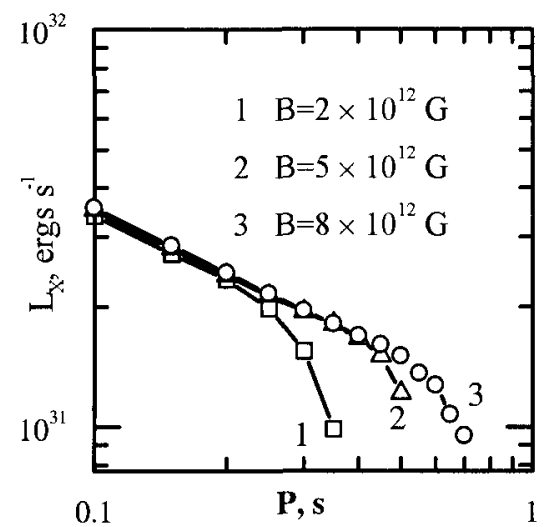

Fig. 3

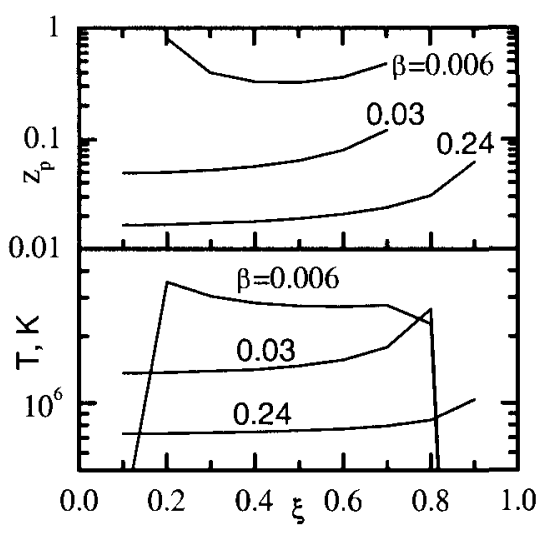

Fig. 4

This work has been partly supported by RFBR (grant 98-02-18401), Russian State Program "Astronomy", and INTAS (grant 96-0154).

\section{References}

Arons J., \& Scharlemann E. T. 1979, ApJ, 231, 854

Beskin V. S. 1990, Soviet Astr.Lett., 16, 286

Goldreich P., \& Julian W. H. 1969, ApJ, 157, 869

Harding A. K., \& Muslimov A. G. 1998, ApJ, 500, 862

Jones P. B. 1985, Phys.Rev.Lett, 55, 1338

Muslimov A. G., \& Harding A. K. 1997, ApJ, 485, 735

Muslimov A. G., \& Tsygan A. I. 1990, Soviet Ast., 34, 133

Muslimov A. G., \& Tsygan A. I. 1991, in IAU Colloq. 128, The Magnetosphere Structure and Emission Mechanisms of Radio Pulsars, ed. Hankins, T., Rankin, J., 248

Muslimov A. G., \& Tsygan A. I. 1992, MNRAS, 255, 61

Neuhauser, D., Koonin, S. E. \& Langanke, K. 1987, Phys.Rev.A, 36, 4163

Pal'shin V. D., \& Tsygan A. I. 1997, Astronomy Reports, 41, 516

Pal'shin V. D., \& Tsygan A. I. 1996, Astronomy Reports, 40, 385

Pal'shin V. D., \& Tsygan A. I. 1998, preprint PTI 1718, St.Petersburg

Pal'shin V. D., \& Tsygan A. I. 1999, in preparation

Ruderman, M., \& Sutherland, P. G 1975, ApJ, 196, 51

Sturrock P. A. 1971, ApJ, 164, 529

Tsygan, A. I. 1997, MNRAS, 292, 317 